\title{
A Model to Optimize University Course Timetable Using Graph Coloring and Integer Linear Programming
}

\author{
M.T.M. Perera, G.H.J. Lanel \\ Department of Mathematics, University of Sri Jayewardenepura
}

\begin{abstract}
This paper presents the design and construction of a faculty course timetable. The system uses an Integer Linear Programming model which attempts to assign groups of course units to time periods where each group is a result of a graph coloring approach. Limited number of lecture halls, large number of subject combinations and growing number of student registration have made the problem very tight which results thousands of variables and constraints to the model. The quality of the solution depends on the location of the time period assigned to the set of course units. Hence the objective function is defined to optimize the allocation of time periods to course units. The model results a feasible solution which has reduced the maximum idle time of students to three hours and it can be implemented with the lecture halls currently available in the faculty of Applied Sciences, University of Sri Jayewardenepura. The model is flexible and allows to change the constraints depending on the faculty requirements and other factors and if necessary, construct alternative schedules.
\end{abstract}

Keywords: Course Timetabling, Graph Coloring, Integer Linear Programming

\section{Introduction}

Timetabling problem involved in allocation of certain resources such as people, rooms or vehicles to a given set of objects as jobs, time periods, routes, etc. such a way that it will satisfy some objectives subject to some constraints. Among those, the university course timetabling problem looks for the best schedule, according to some criteria, in which every element in a set of resources, which may contain lecturers, groups of students, classrooms or laboratories. A set of constraints defines the terms of availability of the different components, so determining the schedule rules, that is, how there sources must be allocated [11]. Not like in a school, a university course unit may followed by different groups of students, hence two course units can have common students, and room availability and their capacity become constraints to the problem.

The timetabling process is long and contains many stages before placing courses into time slots. One needs to reduce a given timetabling problem to a mathematical model, which can then be solved. Hence to automate the university course timetable, one must consider this entire process. The constraints are usually divided into two categories, hard constraints and soft constraints. Hard constraints are strictly imposed. A feasible timetable must satisfy those hard constraints defined by the user. Soft constraints are desirable but not absolutely essential. In real world situations it is, of course, usually impossible to satisfy all soft constraints [2]. A feasible timetable is optimal in the level of satisfaction of the soft constraints. Soft constraints depend on the institutional requirements. The Faculty of Applied Sciences (FAS) at University of Sri Jayewardenepura (USJP) does not possess an automated timetabling system, as in the most academic institutions in Sri Lanka. At the beginning of each academic semester the management and the technical staff is doing minor changes in the predesigned time table in order to meet the new requirements.

The undergraduate program at FAS consists three years of studies for the general degree program and four years of studies for the special degree program and offers fifteen different subjects with twenty four subject combinations each composed with three different subjects for the general degree. In each subject, there are compulsory course units and electives. Students have the freedom to select electives to cover up the credit requirements. There are about 200 course units being offered by the nine departments in the faculty in each semester with limited number of lecture halls, in which there are about 17 lecture halls available in the faculty with different capacities which can mainly divided in to three sizes as large, medium and small.

From Monday to Friday, from $8.00 \mathrm{a} . \mathrm{m}$. to $6.00 \mathrm{p} . \mathrm{m}$. there are about 45 lecturing hours per week. Depending on the credit value, some course units require two consecutive hours of lectures per week while some other require one hour. Some of the courses require practical sessions of two to three hours per week with minimum capacities of laboratories. Due to such limitations, students are grouped and several practical sessions are repeated over the week.

This study intends to develop a model which assigns each course unit to its most desirable time period and then generate a complete conflicts free semester timetable which can be implemented with the available resources in FAS as mentioned above. The main objective of the study is to minimize the wastage of resources mainly, the time of students, lecturers and the administrative staff and minimize the utility consumption. 


\section{Materials and Methods}

As mentioned earlier a model to be designed and then be solved using a mathematical software. Mainly used techniques in the whole process are the graph coloring and the linear programming. The model formulation is based on a grouping of course units, where each course unit in a group is followed by different groups of students and therefore can be scheduled at the same time.

The grouping was done by constructing a graph in which, vertices represent the subjects and an edge between two vertices implies that those subjects are in conflict (i.e. they appeared in a same subject combination). If two subjects appeared in a same combination, a student who falls to that combination must follow the course units offered by both subjects. Hence those course units cannot be scheduled simultaneously. Such conflicts are represented by introducing an edge between them in the corresponding graph.

The graph is then colored using a graph coloring algorithm such that no two vertices sharing a common edge are colored using the same color. Vertex coloring is the most common graph coloring approach. A variety of graph coloring approaches can be found in literature. De Werra shows how to reduce a course timetabling problem to graph coloring approach such as edge coloring and vertex coloring [10]. Rickman and Yellen have constructed a course timetabling system which models the timetabling problem as a vertex coloring problem in a weighted graph [4].

In this case, an initial coloring was applied to the graph constructed on different subjects and the results obtained are used to construct the conflict matrix corresponding to all course units offered within a semester. Hence for one semester of each three years of studies, three graphs could be constructed with the help of the conflict matrix. Similar mechanism can be followed for the other semester.

Based on the resulted groups of course units, a binary Integer Linear Programming (ILP) model was then developed, which search for a solution to assign each group to a time period.

\section{The model}

The ILP model is designed based on the graph coloring result and the model is described below, assuming $k$ groups of course units and $t$ number of onehour time periods are available over the week where both $k$ and $t$ are positive integers.

\section{Notation}

$I=\{$ set of course unit groups $\}=\{1,2,3, \ldots, k\}$

$J=\{$ Set of time periods $\}=\{1,2,3, \ldots, t\}$

$H=\left\{h_{i} \mid h_{i}\right.$ is the duration of course units group $\left.i\right\}$

\section{Decision variable}

Let $i \in I$ and $j \in J$ then the decision variable is: $x_{i j}$. Since there are $k$ groups and $t$ time periods, the model defines $k \times t$ decision variables.

\section{Objective function}

$$
x_{i j}=\left\{\begin{array}{rr}
1, & \text { if } i^{\text {th }} \text { course is assigned to } j^{\text {th }} \text { time period } \\
0, & \text { otherwise }
\end{array}\right.
$$

The ILP model is formulated as a minimization problem and the objective has been designed to minimize the undesirability of assigning a set of course units to a time period and can be defined as follows.

$$
\text { minimize } z=\sum_{i=1}^{k} \sum_{j=1}^{t} x_{i j} p_{j}
$$

The coefficient $p_{j}$ has been determined to reflect the desirability of the assignment of a group of course units to the time period $j$. Higher $p_{j}$ values means less desirability to assign a group to $j^{\text {th }}$ period. For the three models based on the academic year, three values for each period $j$ have been assigned. Table 1 shows the table of coefficients for the first year time periods. The highest priority is given for the first hour of the day and second priority for the second hour. But for the second year timetable, highest priority is given for the third and fourth hours of the day, in the purpose of reducing the conflict for the lecture halls. Because a group may consists four or five course units, if such two groups have been assigned to the same period it would compete for the lecture halls. 


\begin{tabular}{|c|c|c|c|c|c|}
\hline Time & Monday & Tuesday & Wednesday & Thursday & Friday \\
\hline $8.00-8.50$ & 1 & 2 & 3 & 4 & 5 \\
\hline $8.55-9.45$ & 6 & 7 & 8 & 9 & 10 \\
\hline $10.15-11.00$ & 11 & 12 & 13 & 14 & 15 \\
\hline $11.10-12.00$ & 16 & 17 & 18 & 19 & 20 \\
\hline $1.00-2.00$ & 21 & 22 & 23 & 24 & 25 \\
\hline $2.00-3.00$ & 26 & 27 & 28 & 29 & 30 \\
\hline $3.00-4.00$ & 31 & 32 & 33 & 100 & 35 \\
\hline $4.00-5.00$ & 36 & 37 & 100 & 100 & 100 \\
\hline $5.00-5.45$ & 41 & 42 & 100 & 100 & 100 \\
\hline
\end{tabular}

Table 1: Coefficients of the objective function for the first year

The faculty has left the last three hours of every Thursday for students' activities where lectures cannot be scheduled so that, highest $p_{j}$ value has been assigned for those periods in each of the three models.

\section{Set of Constraints}

The problem is solved subject to the following set of constraints. These are the hard constraints of the model which are highly imposed to be satisfied by any feasible solution.

$$
\sum_{j=1}^{t} x_{i j}=h_{i}, \quad \forall i \in I \rightarrow C_{1}
$$

Set of constraints $C_{1}$ assigns each group of course units $i$ to exactly $h_{i}$ number of consecutive time periods. However the requirement of consecutive periods will be addressed later. This would define $k$ number of constraints.

$$
\sum_{i=1}^{k} x_{i j} \leq 1, \quad \forall j \in J \rightarrow C_{2}
$$

$C_{2}$ ensures the assignment of at most one group to a one time period. This will automatically check for a conflict free solution and required $t$ constraints.

$$
x_{i j}-x_{i j+1}=0 \quad \forall i \in I \wedge h_{i}>1 \rightarrow C_{3}
$$

The set of constraints $C_{3}$ is for the consecutive hours, where it will assign $h_{i}$ consecutive time periods for every group $i$ which require more than one hour per week.

$$
0 \leq x_{i j} \leq 1, \forall i \in I \text { and, } \forall j \in J \rightarrow C_{4}
$$

$C_{4}$ is to restrict the solution to positive values in between 0 and 1 , while $C_{5}$ ensures the integer solutions.

$$
x_{i j} \text { is an integer } \forall i \in I \text { and }, \forall j \in J \rightarrow C_{5}
$$

For a single model, it requires $t \times k$ decision variables and hence would results $t \times k$ binary integer solutions. Finally, three models have been designed to three years of studies independently. 


\section{Results and Discussion}

First Semester course unit timetable of FAS, USJP has been modeled and optimal solutions were found for the three years of studies. The data was collected from the faculty prospectus 2015 and the faculty record room. Graph coloring was done using a greedy algorithm with the help of Maple 12. A graph with 15 vertices which represents the 15 subjects offered in FAS, and 38 edges could be constructed initially to categorize the non-conflicting subjects into groups which results five groups.

The different subjects offered in the faculty are, Chemistry (CHE), Mathematics (MAT), Physics (PHY), Computer Science (CSC), Statistics (STA), Management Science (MAN), Information and Communication Technology (ICT), Zoology (ZOO), Aquatic Resource Management (ARM), Plant Bio Technology (PBT), Food Science and Technology (FSC), Polymer Science and Technology (PST), Biology (BIO), Forestry and Environmental Science (EMF) and Economics (ECN). Figure 1 shows the resulted graph coloring.

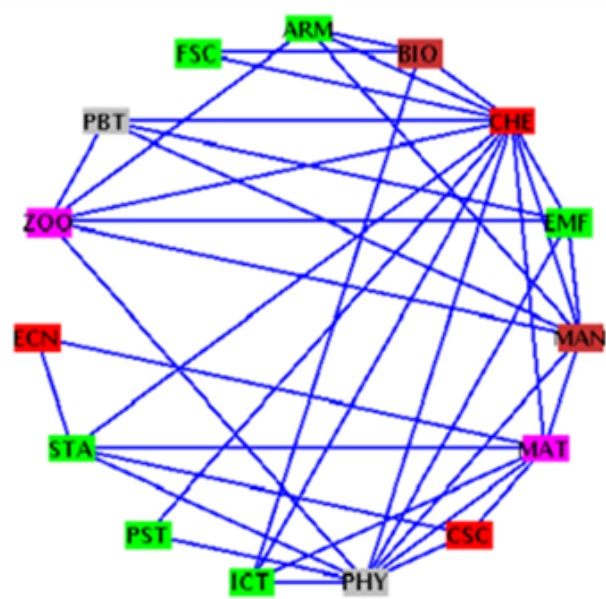

Figure 1: Initial coloring

For each and every subject there are several course units for each year of study. Using the resulted coloring, another three graphs were constructed in Maple 12 using all the course units for each first, second and third year by defining the corresponding conflict matrix and then the problem could be further simplified. For the first year, graph coloring algorithm resulted 20 groups and for the second and third years there are 23 and 24 groups respectively. The set of groups only for the first year is given in Table 2.

\begin{tabular}{|c|c|c|c|}
\hline 1. & $\begin{array}{l}\text { CHE } 1021.0 \text { CSC } 1051.0 \\
\text { ECN } 1011.0\end{array}$ & 11. & ZOO 1261.0 \\
\hline 2. & CHE 1101.0 & 12. & ZOO 1281.0 \\
\hline 3. & CHE 1121.0 & 13. & $\begin{array}{l}\text { CHE } 1072.0 \text { CSC } 1072.0 \\
\text { ECN } 1022.0\end{array}$ \\
\hline 4. & $\begin{array}{l}\text { BIO } 1031.0 \text { MAN } 1041.0 \\
\text { STA } 1151.0 \text { PST } 1041.0\end{array}$ & 14. & $\begin{array}{l}\text { CHE } 108 / 110 \text { PST } 1022.0 \\
\text { CSC } 1061.5\end{array}$ \\
\hline 5. & $\begin{array}{l}\text { ARM } 1011.0 \text { FSC } 1222.0 \\
\text { EMF } 1031.0 \text { ICT102 } 2.0 \\
\text { PST } 1022.0\end{array}$ & 15. & $\begin{array}{l}\text { BIO 102 2.0 MAN } 1022.0 \\
\text { STA } 1142.0 \text { PST } 1012.0\end{array}$ \\
\hline 6. & $\begin{array}{l}\text { ARM } 102 \text { 1.0 FSC } 1111.0 \\
\text { ARM } 1021.0 \text { FSC } 1111.0 \\
\text { PST } 1011.0\end{array}$ & 16. & $\begin{array}{l}\text { BIO } 101 \text { 1.0 MAN } 1012.0 \\
\text { STA } 1132.0 \text { PST } 1021.0\end{array}$ \\
\hline 7. & $\begin{array}{l}\text { ARM } 103 \text { 1.0 FSC } 1211.0 \\
\text { EMF } 1131.0 \text { ICT } 1031.0 \\
\text { PST } 1031.0\end{array}$ & 17. & ARM 1071.0 PBT 1212.0 \\
\hline 8. & $\begin{array}{l}\text { ARM } 104 \text { 1.0 FSC } 1911.0 \\
\text { EMF } 101 \text { 1.0 ICT } 1041.0\end{array}$ & 18. & $\begin{array}{l}\text { PBT } 1222.0 \text { PHY } 104 / 105 \\
\text { PHY } 1032.0\end{array}$ \\
\hline 9. & $\begin{array}{l}\text { ARM } 106 \text { 1.0 EMF } 1151.0 \\
\text { MAT } 1031.0\end{array}$ & 19. & MAT 1012.0 \\
\hline 10. & PBT104 1.0 PHY 1311.0 & 20 & MAT $1022.0 \mathrm{ZOO} 118 / 120$ \\
\hline
\end{tabular}

Table 2: First year course units groups

Using groups of course units, a binary ILP model has been defined for each of the three years and solved with MATLAB 14, since the models result thousands of variables and constraints. The ILP model for the first year is given below based on the resulted 20 groups of the graph coloring and 45 time periods over the week. 
Subject to;

$$
\text { minimize } z=\sum_{i=1}^{20} \sum_{j=1}^{45} x_{i j} p_{j}
$$

$$
\begin{gathered}
\sum_{j=1}^{45} x_{i j}=h_{i}, \quad \forall i \in I \\
\sum_{i=1}^{20} x_{i j} \leq 1, \quad \forall j \in J \\
x_{i j}-x_{i j+1}=0 \quad \forall i \in I \wedge h_{i}>1 \\
0 \leq x_{i j} \leq 1, \quad \forall i \in I \text { and }, \quad \forall j \in J
\end{gathered}
$$

The set of constraints with the binary integer properties of the decision variables and the objective function have been given as the input for a ILP model in MATLAB 14 which uses the Branch and Bound algorithm to construct a sequence of sub problems to search systematically for the optimal solution. This algorithm solves LP relaxations with restricted ranges of possible values of the integer variables. It attempts to generate a sequence of updated bounds on the optimal objective function value. With the solution generated by the model, backtracking was done with some conditional statements since the solution has assigned some groups to $4^{\text {th }}$ and

\begin{tabular}{|c|c|c|c|c|c|}
\hline Time & Monday & Tuesday & Wednesday & Thursday & Friday \\
\hline $8.00-8.50$ & CHE 1101.0 & $\begin{array}{l}\text { BIO 103 } 1.0 \\
\text { MAN } 1041.0 \\
\text { STA } 1151.0 \\
\text { PST } 1041.0\end{array}$ & $\begin{array}{l}\text { CHE } 1072.0 \\
\text { CSC } 1072.0 \\
\text { ECN } 1022.0\end{array}$ & $\begin{array}{l}\text { ARM } 1011.0 \\
\text { FSC } 1222.0 \\
\text { EMF } 1031.0 \\
\text { ICT102 } 2.0 \\
\text { PST 102 } 2.0\end{array}$ & $\begin{array}{l}\text { CHE } 108 / 110 \\
\text { CSC } 1061.5\end{array}$ \\
\hline $8.55-9.45$ & $\begin{array}{l}\text { ARM } 1061.0 \\
\text { EMF } 1151.0 \\
\text { MAT } 1031.0\end{array}$ & & $\begin{array}{l}\text { CHE } 1072.0 \\
\text { CSC } 1072.0 \\
\text { ECN } 1022.0\end{array}$ & $\begin{array}{l}\text { ARM1011.0 } \\
\text { FSC 122 2.0 } \\
\text { EMF 103 1.0 } \\
\text { ICT102 2.0 } \\
\text { PST 102 2.0 }\end{array}$ & $\begin{array}{l}\text { CHE } 108 / 110 \\
\text { CSC } 1061.5\end{array}$ \\
\hline $10.15-11.00$ & $\begin{array}{l}\text { MAT } 1022.0 \\
\text { ZOO } 118 / 120\end{array}$ & $\begin{array}{l}\text { PBT } 1222.0 \\
\text { PHY 104/105 }\end{array}$ & MAT 1012.0 & $\begin{array}{l}\text { ARM } 1071.0 \\
\text { PBT } 1212.0 \\
\text { PHY } 1032.0\end{array}$ & $\begin{array}{l}\text { ARM } 1021.0 \\
\text { FSC111 } 1.0 \\
\text { EMF } 1061.0 \\
\text { ICT 101 } 1.0 \\
\text { PST 101 } 1.0 \\
\end{array}$ \\
\hline $11.10-12.00$ & $\begin{array}{l}\text { MAT } 1022.0 \\
\text { ZOO } 118 / 120\end{array}$ & $\begin{array}{l}\text { PBT } 1222.0 \\
\text { PHY 104/105 }\end{array}$ & MAT 1012.0 & $\begin{array}{l}\text { ARM } 1071.0 \\
\text { PBT } 1212.0 \\
\text { PHY } 1032.0\end{array}$ & $\begin{array}{lll}\text { CHE } 102 & 1.0 \\
\text { CSC } 105 & 1.0 \\
\text { ECN } 101 & 1.0 \\
\end{array}$ \\
\hline $1.00-2.00$ & $\begin{array}{llll}\text { BIO } 101 & 1.0 \\
\text { MAN } 101 & 2.0 \\
\text { STA } 113 & 2.0 \\
\text { PST } 102 & 1.0\end{array}$ & CHE 1121.0 & $\begin{array}{l}\text { ARM } 1041.0 \\
\text { FSC } 1911.0 \\
\text { EMF } 1011.0 \\
\text { ICT 104 } 1.0\end{array}$ & $\begin{array}{l}\text { BIO 102 } 2.0 \\
\text { MAN } 1022.0 \\
\text { STA } 1142.0 \\
\text { PST } 1012.0\end{array}$ & ZOO 1261.0 \\
\hline $2.00-3.00$ & $\begin{array}{l}\text { BIO } 1011.0 \\
\text { MAN } 1012.0 \\
\text { STA1132.0 } \\
\text { PST } 1021.0\end{array}$ & $\begin{array}{l}\text { PBT104 1.0 } \\
\text { PHY } 1311.0\end{array}$ & ZOO 1281.0 & $\begin{array}{l}\text { BIO 102 } 2.0 \\
\text { MAN 102 } 2.0 \\
\text { STA 114 2.0 } \\
\text { PST 101 } 2.0 \\
\end{array}$ & \\
\hline \multicolumn{6}{|l|}{$3.00-4.00$} \\
\hline $4.00-5.00$ & & & & & \\
\hline $5.00-5.45$ & & & & & \\
\hline
\end{tabular}
$5^{\text {th }}$ periods of the day which are 11a.m.-12 noon and 1p.m.-2p.m. which are having a break between them. Such groups have been assigned to $5^{\text {th }}$ and $6^{\text {th }}$ periods of the day.

Finally an optimal solution could be found and it takes less than one minute to generate the solution, even the timetabling problem is NP- hard. The model timetable only for the first year is given in Table 3 .

Table 3: Model timetable for the first year

With the model, a student in the first year will not have lectures after 3.00p.m. For the second year students some of them have lectures up to 5.00p.m. only in the Wednesday and in other days all of them have lectures up to 3.00p.m. But with the large number of electives in the third year, the schedule is having lectures up to 5.00p.m. in the first three days of the week. Depending on the subject combination, students have free time in between lectures. With the current timetable some of them are idling about five hours in the faculty. The 
model is able to reduce the maximum idle time to three hours for the first and second years and four hours for the third year students.

Both graph theoretic and ILP approaches have been used in the formulation. For the three years of studies, timetables were modeled separately and finally three of them were joined together to analyze the feasibility. When constructing the timetable for each academic year, the priority was given to the first year timetable. Since the first year students are new to the system and they must be adapted to the environment, more efficient timetable must be issued to them. Next priority is given for the second year students and last priority is given to third year students. Similarly for the second semester of all three years, a model can be built. It was assumed that lecturer will not become a constraint to the problem where allocation of lecturer to course units is a responsibility of the department which the subject is offering. Further it was assumed that lecture halls belong to each department is accessible to all departments. With those assumptions, it was able to model a conflict free efficient timetable for the FAS at USJP.

\section{Conclusions}

The model was able to optimize the idle time of the students by reducing the maximum idle time to three hours. This idle time can be used to schedule practical sessions for them. Further it was able to implement the result with the currently available lecture halls in the faculty, having 3,6 and 8 large, medium and small size lecture halls where maximum number of large size lecture hall required is 3 for each hour and maximum number from the medium and small size lecture halls is 6 for the solution generated by the model. Hence this model helps to utilize both physical and human resources in the faculty. The problem was solved effectively for the first semester which can be extended to the second semester, and it can be used for other faculties as well. However, the size of the problem creates complications in achieving an optimal solution. It is therefore necessary to find a way of decreasing machine time, which has not been discussed here. This study only searched for a feasible and efficient course unit timetable. Basically it was suggested for the optimization of lecturing hours. The analysis revealed that it can be implemented with the available resources, but it does not allocate each course unit to a lecture hall. As a suggestion it would be assigned using an assignment algorithm such as Hungarian algorithm by further analysis.

\section{References}

[1]. E.K. Burke, S. Petrovic, Recent research directions in automated timetabling, European Journal of Operational Research 140 (2002), pp 266-280.

[2]. Enzhe Yu, Ki Seok, A genetic algorithm for a university weekly course timetabling problem, international transaction in operational research, 9(2002), pp 703-717.

[3]. F. Zibran, A multi phase approach to university course timetabling, M.Sc.Thesis , 2007.

[4]. J. Rickman, J. Yellen, Course Timetabling Using Graph Coloring and A.I. Techniques, $10^{\text {th }}$ International Conference of the Practice and Theory of Automated Timetabling, (PATAT), 26-29, 2014.

[5]. M. Dimopoulou, P. Miliotis, Theory and Methodology - Implementation of a university course and examination timetabling system, European Journal of Operational Research 130 (2001), pp 202-213.

[6]. M. Bakr, C. Aksop, A 0-1 integer programming approach to a university timetabling problem, Hacettepe Journal of Mathematics and Statistics, Volume 37 (1) (2008), pp 41-55.

[7]. Phillips, D. Ryan, Solving the Classroom Assignment Problem Using Integer Programming, University of Auckland, New Zealand, 2013.

[8]. S. Daskalaki, T. Birbas, E. Housos, An integer programming formulation for a case study in university timetabling, European Journal of Operational Research 153 (2004), pp 117-135

[9]. Stephen Chacha, Mathematical programing formulations for optimization of university course timetabling problem, The Case of Makwawa University College of Education, M.Sc. (Mathematical Modeling) Dissertation, University of Dares Salaam September, 2012 .

[10]. T. Maijller, Constraint-based Timetabling, Ph.D. Thesis, Faculty of Mathematics and Physics, Charles University in Prague, 2005.

[11]. Tim B. Cooper and Jefferey H. Kingston, The Solution of Real Instances of the Timetabling Problem, The Computer Journal vol. 36, no.7. Sydney, Australia, 1993. 